\title{
Regulation of the cell cycle gene, BTG2, by miR-21 in human laryngeal carcinoma
}

\author{
Min Liu ${ }^{1}$, Haidong $\mathrm{Wu}^{1}$, Tao Liu ${ }^{1}$, Yixuan $\mathrm{Li}^{1}$, Fang $\mathrm{Wang}^{1}$, Haiying $\mathrm{Wan}^{1}, \mathrm{Xin}^{1}{ }^{1}$, Hua Tang ${ }^{1}$ \\ ${ }^{I}$ Tianjin Life Science Research Center and Basic Medical School, Tianjin Medical University, Tianjin 300070, China
}

\begin{abstract}
MicroRNAs are short regulatory RNAs that negatively modulate gene expression at the post-transcriptional level, and are deeply involved in the pathogenesis of several types of cancers. To investigate whether specific miRNAs and their target genes participate in the molecular pathogenesis of laryngeal carcinoma, oligonucleotide microarrays were used to assess the differential expression profiles of microRNAs and mRNAs in laryngeal carcinoma tissues compared with normal tissues. The oncogenic miRNA, microRNA-21 (miR-21), was found to be upregulated in laryngeal carcinoma tissues. Knockdown of $m i R-21$ by specific antisense oligonucleotides inhibited the proliferation potential of HEp-2 cells, whereas overexpression of miR-21 elevated growth activity of the cells, as detected by the colony formation assay. The cell number reduction caused by miR-21 inhibition was due to the loss of control of the G1-S phase transition, instead of a noticeable increase in apoptosis. Subsequently, a new target gene of miR$21, B T G 2$, was found to be downregulated in laryngeal carcinoma tissues. BTG2 is known to act as a pan-cell cycle regulator and tumor suppressor. These findings indicate that aberrant expression of miR-21 may contribute to the malignant phenotype of laryngeal carcinoma by maintaining a low level of $B T G 2$. The identification of the oncogenic miR-21 and its target gene, $B T G 2$, in laryngeal carcinoma is potentially valuable for cancer diagnosis and therapy.
\end{abstract}

Keywords: microRNA, cell cycle, BTG2, laryngeal carcinoma, microRNA-21

Cell Research (2009) 19:828-837. doi: 10.1038/cr.2009.72; published online 23 June 2009

\section{Introduction}

MicroRNAs (miRNAs) are a group of non-coding, single-stranded RNAs that are approximately $22 \mathrm{nt}$ in length. They regulate gene expression at the post-transcriptional level, and are involved in the regulation of cellular differentiation, development and metabolic processes. Hundreds of miRNAs have been identified in various mammalian genomes. To date, there have been 706 miRNA genes described in the human genome. It is becoming apparent that miRNAs have the potential to regulate at least $20 \%-30 \%$ of all human genes [1], but their biological function remains largely unknown. Recently, researchers have uncovered both the tumor-suppressive

Correspondence: Hua Tang

Tel/Fax: +86-22-23542503

E-mail: htang2002@yahoo.com

Abbreviations: ASO (antisense oligonucleotide); BTG2 (B-cell translocation gene 2); miR-21 (microRNA-21); UTR (untranslated region)

Received 14 May 2008; revised 19 February 2009; accepted 12 May 2009; published online 23 June 2009 and oncogenic potential of a number of miRNAs, underscoring their importance in human cancers [2-4]. In fact, either a single miRNA or small sets of miRNAs have been demonstrated to be dysregulated in diverse cancer subtypes. Aberrant expression of miRNAs can contribute to tumor development and progression by targeting certain tumor suppressor genes. Tumor suppressors maintain cell cycle checkpoint integrity and regulate apoptotic responses, and the deregulation of tumor suppressor genes by miRNAs can enhance tumorigenesis and contribute to poor clinical outcomes. Mertens-Talcott et al. [5] have reported that $m i R-27 a$ facilitates cancer cell proliferation through the suppression of $M y t-1$, which blocks the cell cycle transition at G2-M. Moreover, $m i R-221$ and $m i R$ 222 have also been found to be oncogenes that control cell cycle progression by inhibiting $p 27^{k i p l}[6,7]$.

Laryngeal carcinoma is one of the most common head and neck cancers. Although early-stage laryngeal cancer is often curable with surgery or radiotherapy, for the majority of patients with the advanced disease, the outcome has not improved dramatically in the last two decades despite therapeutic advances. In order to further improve 
survival rates and treatments, it is important to better understand the mechanisms of carcinogenesis. Despite the fact that considerable efforts have been made in recent years, the molecular mechanism involved in the initiation and progression of laryngeal carcinoma remains largely unknown.

This study utilized an oligonucleotide microarray to examine the differential expression profiles of microRNAs and mRNAs in laryngeal carcinoma tissues compared to their adjacent normal tissues. MicroRNA-21 (miR-21), a known oncogenic miRNA, was found to be overexpressed in laryngeal carcinoma tissues, and suppression of $m i R-21$ inhibited the proliferation of the cells of laryngeal carcinoma cell line, HEp-2, by suppressing cell cycle progression without affecting cell apoptosis. Deregulation of $B T G 2$, which was identified to be a $m i R-21$ target gene, may be responsible for the $m i R-21$ mediated abnormal cellular phenotype.

\section{Results}

Differential miRNA expression between laryngeal carcinoma and normal laryngeal tissues

Using the miRNA microarray containing 210 human miRNA probe sets, we first assessed the miRNA profiles in three paired laryngeal carcinoma and normal laryngeal tissues. A miRNA was considered to be significantly upregulated only if the fold-change was more than 1.5 in the cancer tissues compared with the normal tissues. Likewise, only if a fold-change was less than 0.66 was the miRNA considered to be significantly downregulated. Statistical analysis identified 13 miRNAs that were differentially expressed in all three tumor tissues compared with the matched non-neoplastic controls. Among these 13 miRNAs, 6 miRNAs (let-7a-1, miR-203, miR-205, $m i R-21, m i R-98$ and $m i R-16-1)$ were upregulated and 7 miRNAs (miR-100, miR-1-2, miR-122a, miR-143, miR145 , $m i R-26 a-1$ and $m i R-34 c$ ) were downregulated in laryngeal carcinoma tissues (Table 1). Among them, miR21 is reported to be overexpressed in many types of solid tumors, including glioblastoma and breast cancer [8-11]. Thus, we selected $m i R-21$ for further studies.

\section{The effect of miR-21 on the growth of HEp-2 cells}

To study the biological significance of elevated $m i R$ 21 levels in laryngeal carcinoma cells, we first used a loss-of-function approach by transfecting the laryngeal carcinoma cell line, HEp-2, with chemically synthesized oligonucleotides complementary to $m i R-21$. Transient transfection of HEp-2 cells with miR-21 ASO (specific antisense oligonucleotide of $m i R-21$ ) caused suppression of cellular proliferation at 48 -h and 72 -h post-trans- fection in the MTT (3-(4,5-dimethylthiazol-2-yl)-2,5diphenyltetrazoliumbromide) assay (Figure 1A). Meanwhile, the soft agar colony formation assay indicated that the number of colonies from HEp-2 cells transfected with $m i R-21$ ASO was significantly lower than that of the negative control group (Figure 1B) and that overexpression of $m i R-21$ using a $m i R-21$-expressing construct (pSilencer/sh-miR-21) led to elevated colony formation in tumor cells (Figure 1C). In addition, we also explored the effect of $m i R-21$ on laryngeal tumorigenesis in vivo. Compared with the control group, knockdown of $m i R-21$ led to suppression of overall tumor growth in nude mice (Figure 1D). Furthermore, the validity of the alterations of $m i R-21$ expression levels was confirmed using realtime PCR assays (Figure 1E). These data indicate that the aberrant expression of $m i R-21$ in laryngeal carcinoma may contribute to the malignant phenotype.

Suppression of miR-21 impairs the HEp-2 cell cycle without affecting apoptosis

To explore how the suppression of $m i R-21$ inhibits HEp-2 cell growth, we studied the effects of miR-21 on cell cycle and cell apoptosis. The cell cycle distribution of HEp-2 cells was found to be affected by $m i R-21$.

Table 1 Differentially expressed miRNAs in laryngeal carcinoma tissues compared with normal tissues

\begin{tabular}{llcl}
\hline $\begin{array}{l}\text { miRNA } \\
\text { name }\end{array}$ & Chromosome & $\begin{array}{c}\text { Ratio (cancer/normal } \\
\text { tissue) }\end{array}$ & $P$ value \\
\hline let-7a-1 & 9 & $2.465 \pm 0.831$ & $1.215 \mathrm{E}-04$ \\
$m i R-203$ & 14 & $8.150 \pm 3.816$ & $4.350 \mathrm{E}-04$ \\
$m i R-205$ & 1 & $7.660 \pm 3.665$ & $2.604 \mathrm{E}-07$ \\
$m i R-21$ & 17 & $5.268 \pm 1.756$ & $6.208 \mathrm{E}-06$ \\
$m i R-98$ & $X$ & $4.485 \pm 2.431$ & $4.252 \mathrm{E}-07$ \\
$m i R-16-1$ & 13 & $11.745 \pm 8.842$ & $1.607 \mathrm{E}-03$ \\
$m i R-100$ & 11 & $0.119 \pm 0.038$ & $8.139 \mathrm{E}-06$ \\
$m i R-1-2$ & 18 & $0.126 \pm 0.053$ & $7.727 \mathrm{E}-04$ \\
$m i R-122 a$ & 18 & $0.183 \pm 0.027$ & $1.968 \mathrm{E}-06$ \\
$m i R-143$ & 5 & $0.331 \pm 0.173$ & $1.342 \mathrm{E}-03$ \\
$m i R-145$ & 5 & $0.186 \pm 0.042$ & $9.411 \mathrm{E}-06$ \\
$m i R-26 a-1$ & 3 & $0.390 \pm 0.024$ & $2.718 \mathrm{E}-06$ \\
$m i R-34 c$ & 11 & $0.201 \pm 0.113$ & $6.211 \mathrm{E}-07$ \\
\hline
\end{tabular}

The miRNA microarray analysis was performed to detect the differentially expressed miRNAs in laryngeal carcinoma tissues and matched normal tissues. The prominently changed miRNAs are shown in the figure. The ratio indicates the fold change (mean $\pm \mathrm{SD}$ ) of cancer tissues compared with the normal tissues and the $P$ value is also provided. 

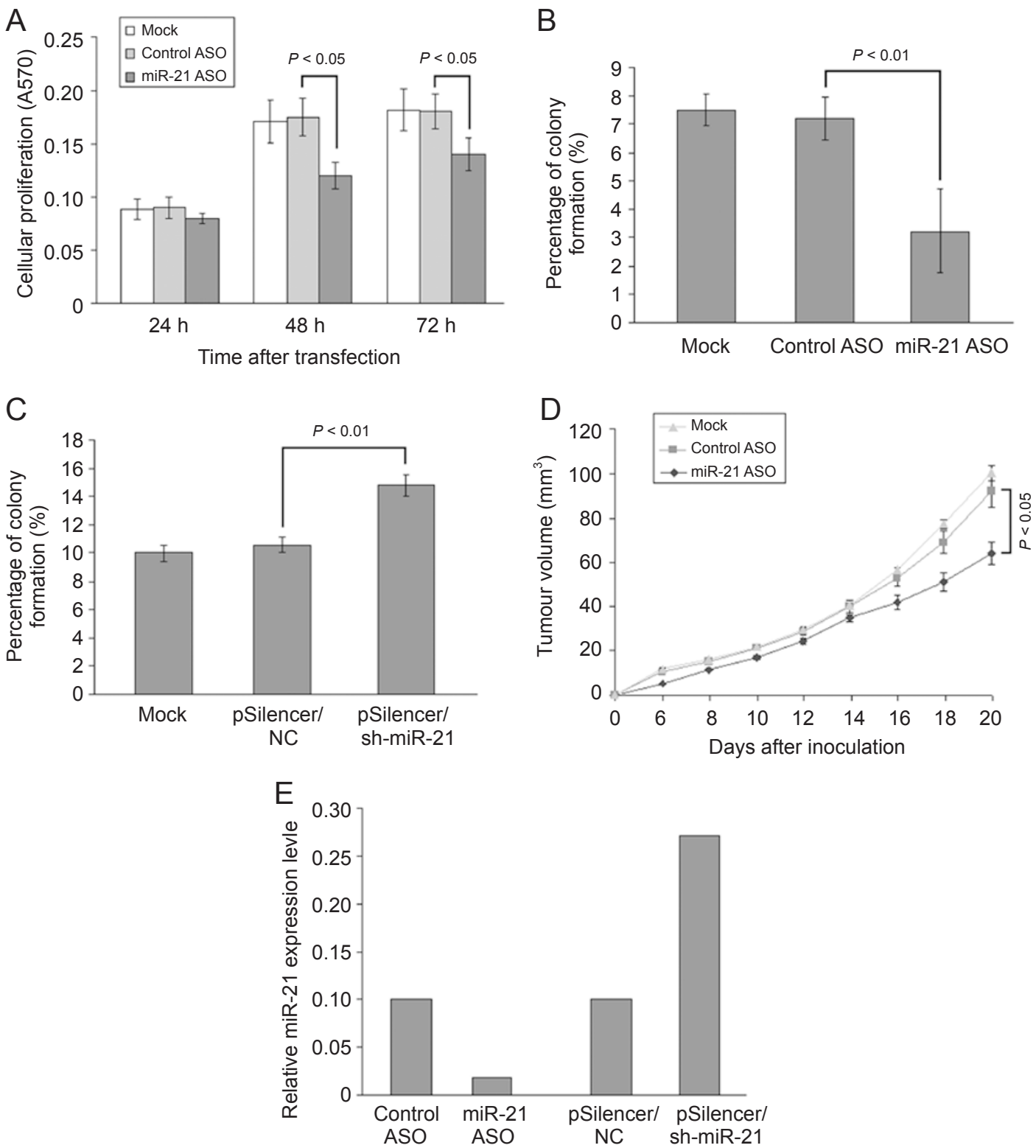

Figure 1 Regulation of tumor cell proliferation by miR-21. (A) HEp-2 cells were either mock transfected, transfected with miR-21 ASO or transfected with control ASO. The MTT assay was used to determine relative cellular proliferation at $24 \mathrm{~h}$, $48 \mathrm{~h}$ and $96 \mathrm{~h} . \mathrm{A}_{570}$ is the optical density of MTT measured at $570 \mathrm{~nm}$. The figure shows mean values $\pm S D$ of $A_{570}$ from three independent experiments. After transfection with miR-21 ASO, the $48 \mathrm{~h}$ and $72 \mathrm{~h}$ data points showed a statistically significant difference. (B) HEp-2 cells were either mock transfected, transfected with miR-21 ASO, or transfected with control ASO. At 48-h post-transfection, tumor cell proliferation was analyzed by a soft agar colony formation assay. (C) pSilencer/sh-miR-21 or control vector (pSilencer/NC) was transfected into HEp-2 cells. At 48-h post-transfection, tumor cell proliferation was analyzed by a soft agar colony formation assay. All the histograms show normalized mean value \pm SD of percentage of colony formation from three independent experiments. (D) Growth curves of primary subcutaneous tumors in nude mice formed by HEp-2 cells either non-transfected or transfected with miR-21 ASO or control ASO. Each data point represents the mean \pm SD of six mice. $P$ value is based on Student's $t$-test at day 20. (E) In HEp2 cells, miR-21 was blocked by miR-21 ASO or was overexpressed by pSilencer/sh-miR-21 vector, and then the miR-21 expression level was detected using real-time RT-PCR assays. The histogram shows the relative miR-21 expression levels.

When the cells were transfected with miR-21 ASO, the number of cells in the G1 phase significantly increased, and G1-S arrest was obvious (Figure 2A). However, there was no significant increase of cell apoptosis in $m i R$ - 
A

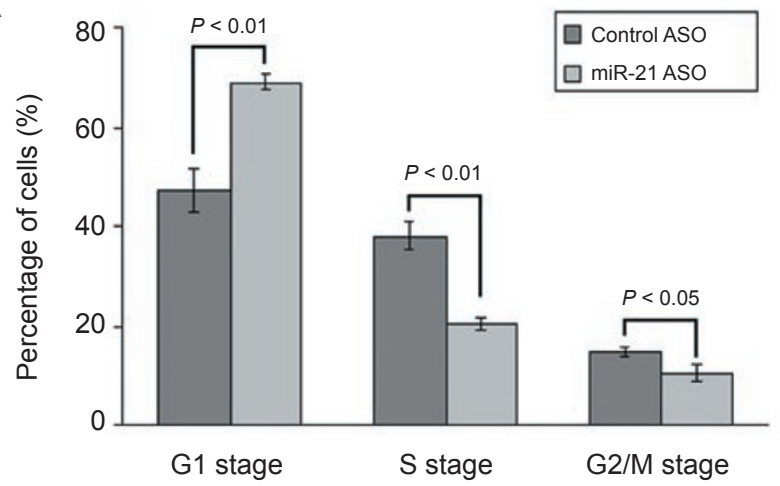

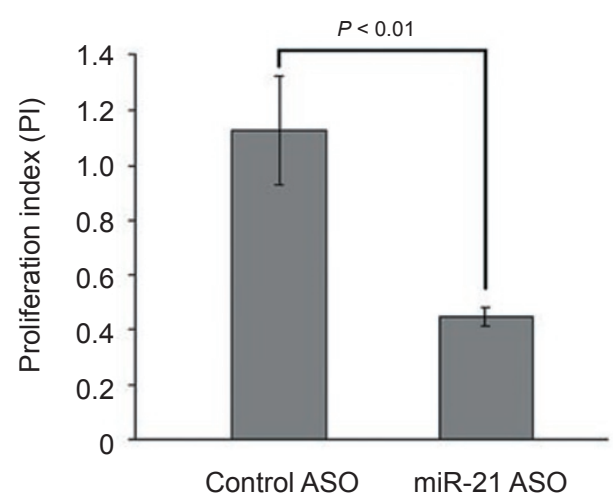

B
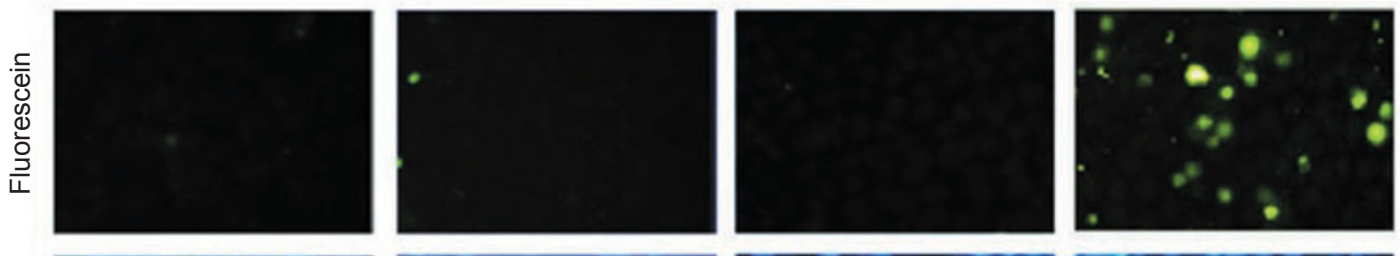

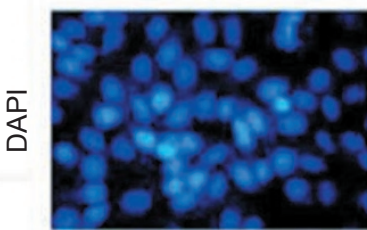

miR-21 ASO

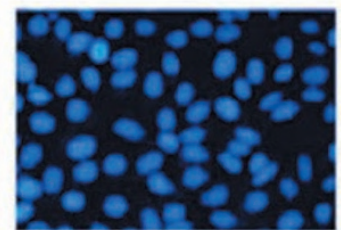

Control ASO

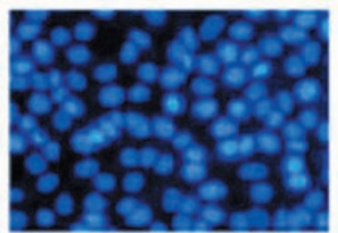

HEp-2 cell

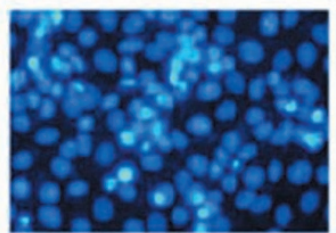

VM-26

Figure 2 The effect of miR-21 suppression on cell cycle and cell apoptosis. (A) At $48 \mathrm{~h}$ after transfection with miR-21 ASO or control ASO, HEp-2 cells were detached, rinsed, fixed and stained. Quantification of the cell cycle phase distribution was analyzed by flow cytometry. The number of cells in the G1 phase was significantly increased in the miR-21 ASO group, and G1-S arrest was obvious. The proliferation index (PI) decreased significantly in the miR-21 ASO group. Data are shown as the mean $\pm S D$ of three replicates and are representative of three independent experiments. (B) HEp-2 cells were transfected with miR-21 ASO or control ASO, and detected by the In situ Cell Death Detection Kit at 48-h post-transfection. Fluoresceinstained cells indicated apoptotic cells. HEp-2 cells treated with $0.09 \mathrm{mg} / \mathrm{ml}$ teniposide (VM-26) were used as positive controls, and untreated HEp-2 cells were used as negative controls. DAPI staining was used to determine the number of nuclei and to assess the gross cell morphology.

21-ASO-transfected cells compared with control-ASOtransfected cells, although there were a few apoptotic cells due to liposome transfection (Figure 2B). These results indicate that the change of cell number in $m i R$ 21-ASO-transfected HEp-2 cells was due to the loss of control of the cell cycle instead of an increase in cell apoptosis. Thus, $m i R-21$ may contribute to laryngeal tumorigenesis by regulating cell cycle checkpoints and cellular proliferation.

BTG2 is downregulated in laryngeal carcinoma and is a putative target of miR-21

MicroRNAs regulate a variety of cellular activities through regulation of the expression of multiple target genes. To understand the mechanism of $m i R$ - 21 -mediated cell cycle dysregulation in laryngeal carcinoma, we next focused on the identification of target genes that may participate in this process. As $m i R-21$ has been validated as being elevated in laryngeal carcinoma, its target genes should be correspondingly downregulated in laryngeal carcinoma. The 7267 -spot human cDNA microarray was used to investigate the gene expression profiles of the three paired laryngeal carcinoma tissues compared with the adjacent normal tissues. As a result, 90 genes were found to be differentially expressed in the laryngeal carcinoma tissue. Among these genes, 29 were upregulated and 61, including $B T G 2$, were downregulated. In order to find $m i R-21$ target genes, we combined the datasets obtained from our microarray analysis with the datasets obtained from publicly available databases. It is well known 
that miRNAs usually bind their targets with incomplete complementarity within the $3^{\prime}$ untranslated region (UTR) of the mRNA target. Most bioinformatics methods use the first 2-8 bases of the mature miRNA sequence to search for sequences complementary to the 3'UTR of all expressed genes. Publicly available databases (PicTar and TargetScan) were used for target predication [12, 13]. BTG2, a pan-cell cycle regulator that is confirmed to be downregulated in laryngeal carcinoma using semiquantitative RT-PCR, was predicted to be a target gene of $m i R-21$ (Figure 3A).
$M i R-21$ regulates $B T G 2$ at both the $m R N A$ and the protein levels

To determine the effect of $m i R-21$ on $B T G 2$ expression levels, total RNA and protein were isolated from HEp-2 cells transfected with $m i R-21$ ASO or control ASO. The mRNA level of BTG2 was quantified using semi-quantitative RT-PCR, and the protein level of $B T G 2$ was detected by western blot. The results showed that both the mRNA and the protein levels of $B T G 2$ were elevated in HEp-2 cells transfected with miR-21 ASO compared with cells transfected with control ASO (Figure
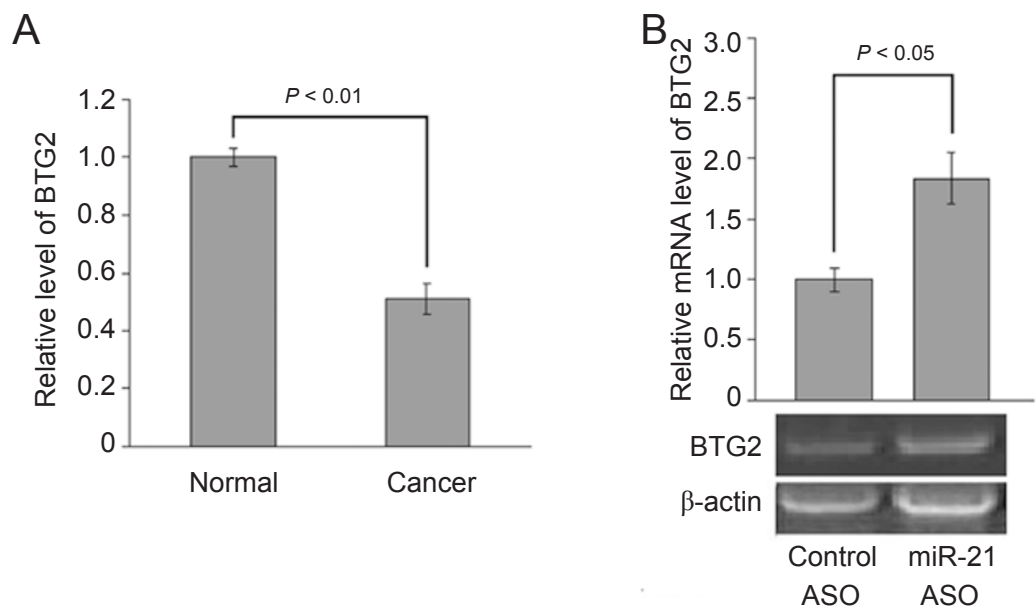
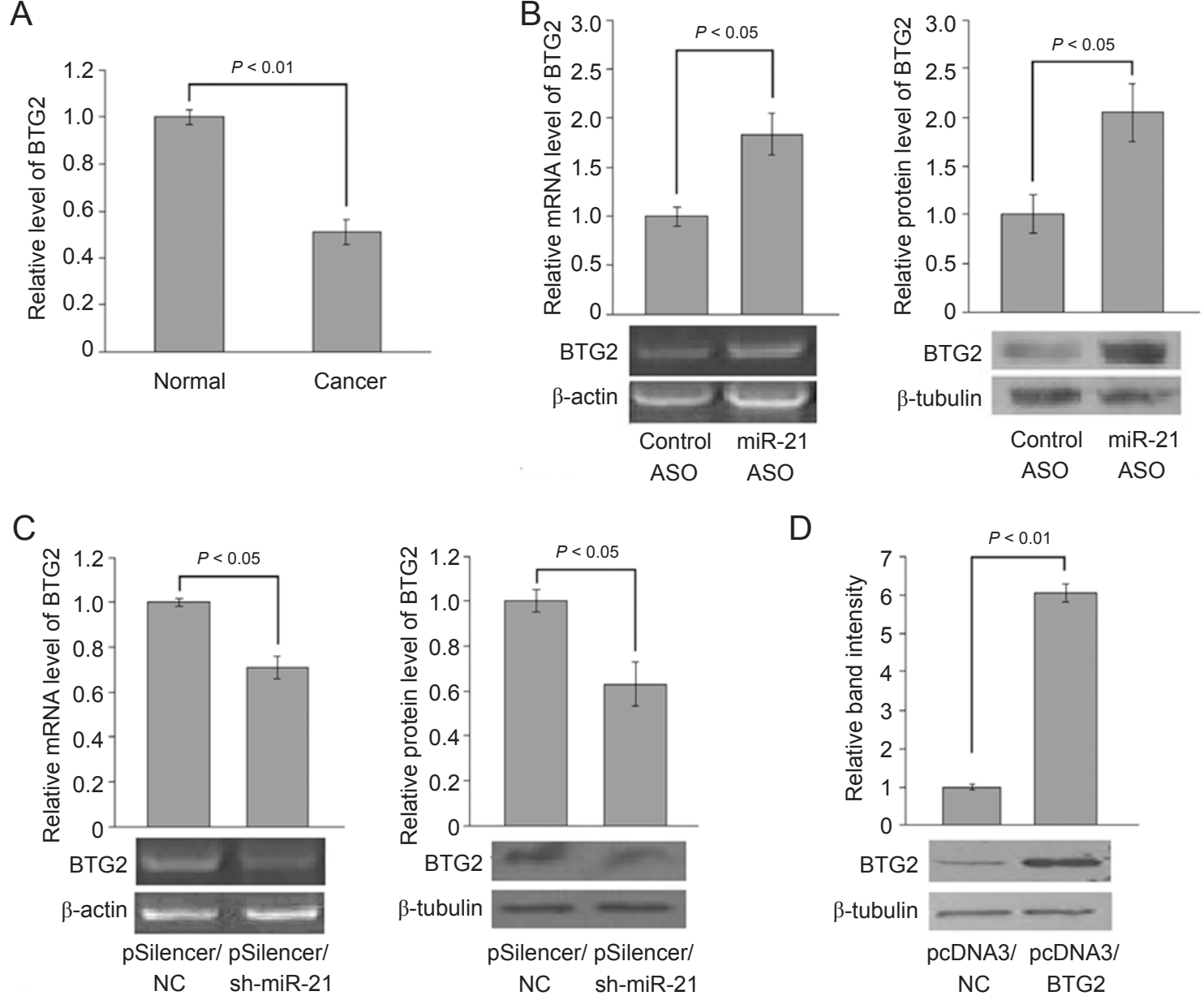

Figure 3 Regulation of BTG2 expression by miR-21. (A) The differential expression of BTG2 in three paired clinical samples from laryngeal carcinoma tissues and adjacent normal tissues were validated using semi-quantitative RT-PCR. The data represents the mean value $\pm S D$, and the normal group was set to 1. (B) HEp-2 cells were transfected with either miR-21 ASO or control ASO. At 48-h post-transfection, the mRNA level of BTG2 was determined by semi-quantitative RT-PCR and the BTG2 protein level was detected by western blot. The histograms show the quantification results for semi-quantitative RT-PCR or western blot. The expression level of BTG2 in the control ASO group was set to 1. (C) pSilencer/sh-miR-21 or control vector was transfected into HEp-2 cells. At 48-h post-transfection, the BTG2 RNA and protein levels were assessed. The expression level of BTG2 in the control group was set to 1. (D) The BTG2 protein expression vector, pcDNA3/BTG2, as well as the control vector was transfected into HEp-2 cells and the BTG2 level of these cells was detected with western blot assay to determine the efficacy of the anti-BTG2 antibody. The expression level of BTG2 in the control group was set to 1. All the data are shown as the mean value \pm SD of three independent experiments. 
3B). Similarly, HEp-2 cells were transfected with either a miR-21-expressing vector (pSilencer/sh-miR-21) or a control vector ( $\mathrm{pSilencer} / \mathrm{NC}$ ). At 48 -h post-transfection, $B T G 2$ RNA and protein levels were assessed. We found that overexpression of $m i R-21$ in HEp-2 cells could reduce the expression of $B T G 2$ at both the mRNA and the protein levels (Figure $3 \mathrm{C}$ ). In addition, when BTG2 protein was overexpressed in HEp-2 cells, a band with higher intensity was detected in western blot, demonstrating the specificity of the anti-BTG2 antibody (Figure 3D).

\section{Direct effect of miR-21 on the 3'UTR of BTG2}

An EGFP (enhanced green fluorescent protein) reporter assay was used to demonstrate that the negative regulation of $m i R-21$ on $B T G 2$ expression was direct. The alignment of $m i R-21$ with the BTG2 $3^{\prime} \mathrm{UTR}$ is illustrated in Figure 4A. The 3'UTR sequence of BTG2 was cloned downstream of the EGFP gene. We found that the expression of EGFP was strongly increased when $m i R$ 21 function was inhibited by transfecting cells with an antisense oligonucleotide (Figure 4B), whereas miR-21 overexpression reduced EGFP expression (Figure 4C). To further substantiate a direct effect of $m i R-21$ on the $B T G 2$ 3'UTR, we introduced a triple mutation in the seed sequence of the BTG2 3'UTR complementary to miR-21. The EGFP expression from the mutated vector, pcDNA3/ EGFP-BTG2MUT, was not affected by $m i R-21$, regardless of its expression levels (Figure 4B and 4C). We thus conclude that $m i R-21$ regulates $B T G 2$ expression directly through the UTR-binding region.

\section{Discussion}

MicroRNAs are a novel class of regulatory molecules with the ability to control gene expression at the posttranscriptional level. MiRNAs appear to decrease protein production either by increasing the degradation of mRNA or by blocking translation of mRNA into protein. Hence, changes in the expression of even a single miRNA may lead to changes in diverse cellular activities, which may contribute to the development of diseases. In the present study, miRNA microarray assay was used to assess the miRNA expression profiles of three laryngeal carcinoma tissues compared to their matched normal tissues. A total of 13 miRNAs were identified to be differentially expressed. Of the six upregulated miRNAs, $m i R-21$ is known as an oncomir It is overexpressed in many types of tumor tissues, and contributes to malignant phenotypes [8-11]. High expression of $m i R-21$ in laryngeal carcinoma tissues may also correlate with tumor development and progression. In our study, suppression of $m i R-21$ in cultured HEp2 cells decreased tumor cell
A

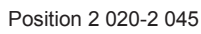

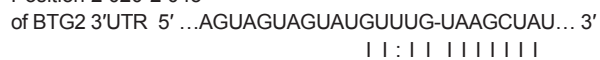

B

miR-21 $3^{\prime} \quad$ AGUUGUAGUCAGACUAUUCGAU $5^{\prime}$

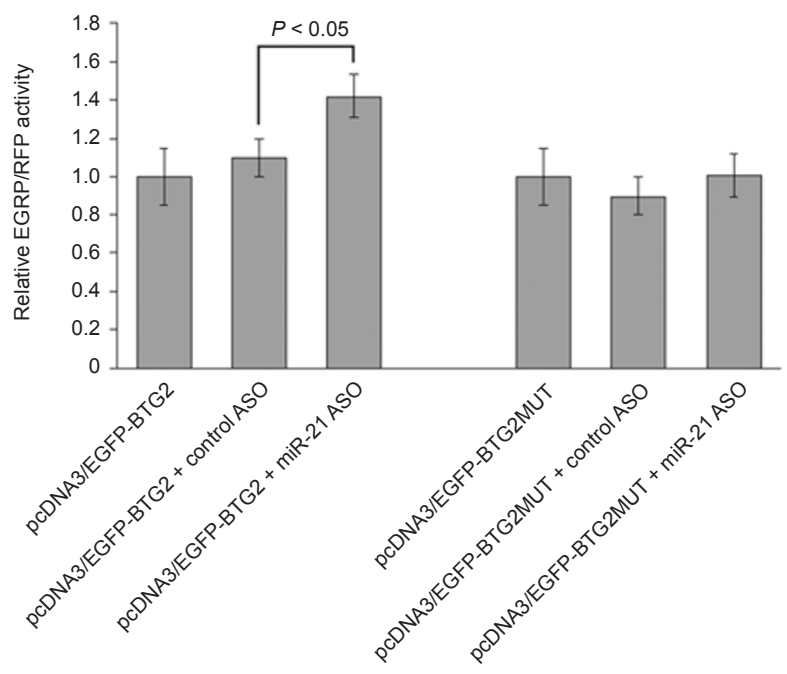

C

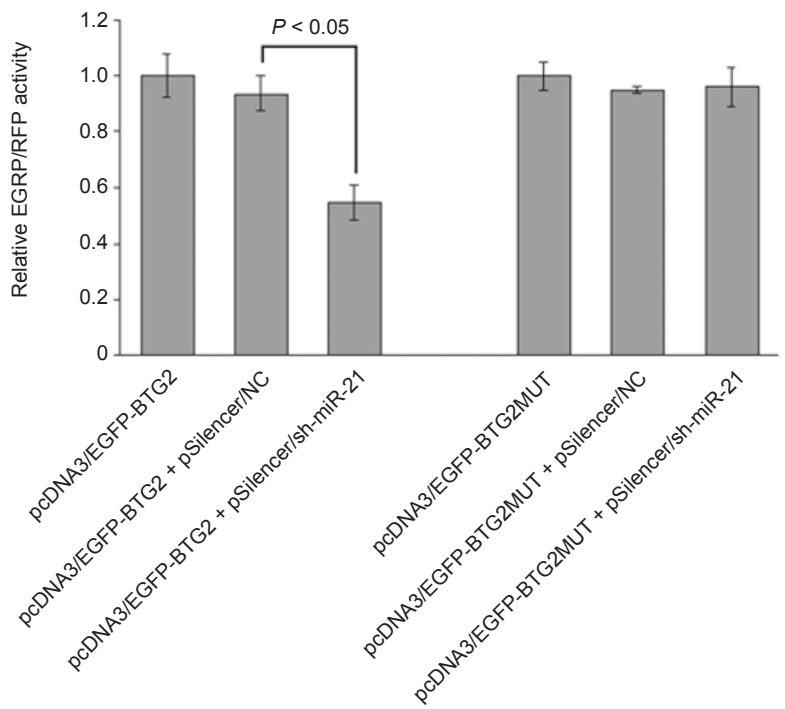

Figure 4 Direct effect of miR-21 on the $3^{\prime} \mathrm{UTR}$ of BTG2. (A) A complementary sequence of $B T G 2$ to miR-21 was obtained from publicly available algorithms. (B) HEp-2 cells were cotransfected with an EGFP reporter plasmid (pcDNA3/EGFPBTG2 or pcDNA3/EGFP-BTG2MUT) and a pDsRed2-N1 plasmid, either alone or together with oligonucleotides. The fluorescence value in the control group was set to 1. (C) HEp-2 cells were co-transfected with EGFP reporter plasmid and pDsRed2N1 plasmid, either alone or together with a miRNA-expressing vector. At $48 \mathrm{~h}$ after transfection, EGFP and RFP activities were measured with an F-4500 fluorescence spectrophotometer. The fluorescence value in the control group was set to 1 . All the histograms show normalized mean values $\pm S D$ of fluorescence intensity from three independent experiments.

proliferation in vitro and in vivo, whereas overexpression of $m i R-21$ elevated the proliferation potential. The 
growth suppression by $m i R-21$ inhibition was found to be due to the loss of control of the G1-S phase transition, and not due to an increase in cell apoptosis, as shown by flow cytometry and cell apoptosis assay.

It is now well known that miRNAs regulate a variety of cellular activities through their effect on the expression of multiple target genes [14]. Identification of the target genes of miRNAs may help to characterize the diverse functions of miRNAs. Recent reports have found that PTEN, TPM1 and PDCD4 are target genes of $m i R$ 21 [15-18]. However, given that a single miRNA has multiple targets, we believe that $m i R-21$ also has other targets. Target prediction and validation are still challenges in miRNA research. Since the regulation of gene expression at the mRNA level may be a common mechanism for miRNA function, it is convenient to monitor transcriptional changes using a high-throughput approach such as a microarray [19]. Combining target prediction and expression profiling has been shown to be effective [20]. According to the results of the gene expression array from the three paired normal and cancer tissues, 29 genes were upregulated and 61 genes were downregulated in laryngeal carcinoma. Furthermore, the previously confirmed targets of $m i R-21$, including PTEN, $T P M 1$ and $P D C D 4$, also showed a downregulation trend in at least one of the cDNA microarrays, demonstrating that microarray analysis is a valid method to help find miRNA targets. As a new candidate target gene of $m i R$ $21, B T G 2$ is downregulated in laryngeal carcinoma, and meanwhile, it is predicted to be a target gene of $m i R-21$ according to publicly available databases.

Several results indicate that $B T G 2$ is a target of $m i R$ 21. First, the 3 'UTR of $B T G 2$ contains a sequence matching the $m i R-21$ seed region. Second, suppression of miR-21 caused enhanced expression of a BTG2 3'UTRcontaining fluorescent reporter in HEp2 cells, whereas $m i R-21$ overexpression reduced the EGFP level. Third, a site mutation in the BTG2 $3^{\prime}$ UTR complementary to $m i R$ 21 abolished $m i R-21$ regulation. Finally, the mRNA and protein levels of $B T G 2$ in HEp-2 cells were specifically correlated with the expression level of $m i R-21$. An interesting fact is that the extent of change of BTG2 protein is more obvious than that of $B T G 2$ mRNA. A possible explanation is that aside from mRNA degradation, the transcriptional repression mechanism also contributes to the regulation of $B T G 2$ by $m i R-21$. Recently, using array expression analysis, Frankel et al. [17] also found upregulation of $B T G 2$ in MCF-7 cells depleted of $m i R-21$. In short, $m i R-21$ regulates $B T G 2$ expression directly by binding to its $3^{\prime} \mathrm{UTR}$.

The $B T G 2$ belongs to an anti-proliferative gene family, which is highly conserved among various species.
Many potential roles of $B T G 2$ have been suggested, such as a transcriptional co-regulator, a differentiation and anti-apoptotic factor in neurogenesis, a key mediator of the stage-specific expansion of thymocytes, a negative regulator of hematopoietic progenitor expansion, and a tumor suppressor in both mice and humans [21]. It has been found that the expression of BTG2 is constitutive and high in the thymus, lung alveolar epithelium, proximal tubule of the kidney and basal cell layer of prostate acini, whereas the mRNA level of $B T G 2$ is usually low in their corresponding tumors [21-25]. Our research also validated the downregulation of BTG2 in laryngeal carcinomas by microarray and semi-quantitative RT-PCR. The loss of $B T G 2$ expression may play an important role in the carcinogenic process. Aberrant expression of $m i R$ 21 may account for the repression of $B T G 2$ levels in laryngeal carcinoma, although other mechanisms cannot be excluded.

In addition, $B T G 2$, as a pan-cell cycle regulator, induces G1-S arrest via $p R B$-dependent and $p R B$-independent pathways $[26,27]$. The former is carried out through the inhibition of cyclin D1 expression, and the latter by delayed biosynthesis of cyclin E and CDK4 proteins [21, $26,27]$. Thus, the identification of $B T G 2$ as a $m i R-21$ target gene provides a possible explanation as to why the suppression of $m i R-21$ can inhibit the growth of tumor cells through influencing their cell cycles. As low $p R B$ expression is frequently found in laryngeal carcinoma, suppression of miR-21 may induce G1-S arrest, mainly through a $p R B$-independent pathway, by upregulating the levels of $B T G 2[27,28]$. However, further study is necessary to gain a full understanding of the underlying molecular mechanism.

Recently, aberrant expression of $m i R-21$ has been found to contribute to the malignant phenotype by blocking the expression of critical apoptosis-related genes $[10,11]$. Our research indicates that miR-21 suppression inhibits the growth of HEp2 cells without affecting apoptosis. Thus, loss of cell cycle control may be the potential mechanism of miR-21-mediated tumorigenesis in laryngeal carcinoma. Emerging evidence suggests that some miRNA activities influence cell cycle progression [5-7, 29]. Alteration of miRNA levels can thus contribute to pathological phenotypes by regulating cell cycle-related genes.

In conclusion, $m i R-21$ functions as an oncomir and is overexpressed in laryngeal carcinoma tissues compared to their adjacent normal tissues. Suppression of $m i R-21$ represses cell growth, possibly through inhibition of the cell cycle by targeting $B T G 2$, but not through a marked increase in apoptosis. Thus, the identification of the oncogene, $m i R-21$, and its target gene, $B T G 2$, in laryngeal 
carcinoma may help us to understand the molecular mechanism of tumorigenesis, and may have diagnostic and therapeutic values in the future.

\section{Materials and Methods}

\section{Laryngeal carcinoma specimens, cell lines and isolation of RNAs}

Laryngeal carcinoma specimens were collected from the Tianjin First Center Hospital. The paired fresh samples from tumor tissues and adjacent normal tissues were obtained from patients with sporadic laryngeal carcinoma undergoing surgical resection. The samples were snap-frozen in liquid nitrogen and stored at $-80^{\circ} \mathrm{C}$. The laryngeal carcinoma cell line, HEp-2, was cultured at $37{ }^{\circ} \mathrm{C}$ with $5 \% \mathrm{CO}_{2}$ in RPMI- 1640 media supplemented with $10 \% \mathrm{FBS}$, $100 \mathrm{U} / \mathrm{ml}$ penicillin and $100 \mu \mathrm{g} / \mathrm{ml}$ streptomycin. Total RNA and enriched small RNAs were obtained from the tissues using the mirVana miRNA Isolation Kit (Ambion, Austin, TX, USA) according to the manufacturer's recommendations. Total RNA was extracted from HEp-2 cells using the TRIzol reagent (Invitrogen, CA, USA).

\section{MiRNA microarray analysis}

A total of 210 DNA oligonucleotide probes from the mirVana miRNA Probe Set (Ambion) were designed according to the sequence of their respective mature miRNAs. Probes were resuspended at $50 \mathrm{mM}$ in $3 \times$ saline sodium citrate (SSC) and spotted on MICROMAX Glass Slides SuperChip I (PerkinElmer, MA, USA) in duplicates at $50 \sim 60 \%$ humidity by the SpotArray 24 Microarray Printing System (PerkinElmer). Small RNAs were labeled with $\mathrm{Cy} 5$ or $\mathrm{Cy} 3$ dyes (Amersham Biosciences, Piscataway, NJ, USA) using the mirVana miRNA Labeling Kit (Ambion). After hybridization at $42{ }^{\circ} \mathrm{C}$ overnight $(12-16 \mathrm{~h})$, the slides were washed in SSC, and scanned with a ScanArray Express Microarray Scanner using ScanArray 3.0 software (PerkinElmer).

\section{cDNA microarray analysis}

The mRNAs from the three paired tumor and normal tissues were reverse transcribed using the MICROMAX TSA Labeling and Detection Kit (PerkinElmer), and the resulting cDNAs were labeled with fluorescein or biotin. Labeled cDNAs were purified and hybridized to DNA arrays containing 7267 human genes relating to human cancer, apoptosis, signal transduction and liver enzymes (Qiagen Inc., CA, USA). Data normalization and calculation of expression values were performed using the ScanArray Express Microarray Analysis System (PerkinElmer).

\section{Vector construction}

The $m i R-21$-expressing vector (pSilencer/sh-miR-21) was constructed according to the manufacturer's instructions for $\mathrm{pSi}$ lencer 2.1 neo (Ambion). The plasmid expresses a miRNA hairpin that can be processed by the cellular machinery into mature $m i R$ 21. The negative control pSilencer 2.1 neo vector (pSilencer/NC) expresses a hairpin siRNA with limited homology to all known sequences in the human genome.

To construct the $B T G 2$ expression vector, the fragment of $B T G 2$ (GenBank accession No. NM_006763) coding region was amplified by RT-PCR from the cDNA library of normal laryngeal tissues using primers BTG2 sense, 5'-CGG AAT TCC GCG ACA TGA GCC ACG GGA AG-3'; and BTG2 antisense, 5'-GCA GCT CGA GGC CTA GCT GGA GAC TGC CAT C-3'. The amplified fragment was cloned into pcDNA3 at $E c o$ RI and $X h o I$ sites to form the pcDNA3/BTG2 vector.

To construct the fluorescence reporter plasmids, the EGFP gene from the pEGFP-N2 vector (Clontech, Mountain View, CA, USA) was subcloned into pcDNA3 and this is referred to as pcDNA3/ EGFP. The pcDNA3/EGFP-BTG2 reporter plasmid was constructed as follows: the 3'UTR sequence of $B T G 2$ was obtained by RTPCR from cDNA library of normal laryngeal tissues using specific primers (BTG2 UTR forward: 5'-CTA GGA TCC AGC CTC ATG GTC TC-3'; and BTG2 UTR reverse: 5'-GGC GAA TTC AGT AAC CTT TGT CTT-3') containing the EcoRI and BamHI endonuclease recognition sites. This fragment was cloned into the pcDNA3/EGFP vector at the EcoRI and BamHI sites, downstream of the EGFP gene. We also constructed pcDNA3/EGFP-BTG2MUT, a plasmid with a triple point mutation (TAAGCTA was mutated to TTACGTA) in the seed sequence of pcDNA3/EGFP-BTG2.

\section{Transfection}

The HEp-2 cells were transfected with oligonucleotides or plasmids using Lipofectamine 2000 reagent (Invitrogen) at $24 \mathrm{~h}$ after plating. Transfection complexes were prepared according to the manufacturer's instructions. The final oligonucleotide concentration was $10 \mathrm{nM}$, and the final plasmid concentration was $0.5 \mathrm{mg} /$ 1. The transfection medium was replaced at 4-h post-transfection. The oligonucleotides complementary to miRNAs were synthesized by IDT (Coralville, IA, USA) and their sequences were as follows: $m i R-21$ antisense oligonucleotide (miR-21 ASO): 5'-TCA ACA TCA GTC TGA TAA GCT A-3'; and negative control oligonucleotide (control ASO): 5'-GTG GAT ATT GTT GCC ATC A-3'.

\section{Cell proliferation assay}

After transfection, the HEp-2 cells were seeded in 96-well plates at 5000 cells per well. The MTT assay was used to determine relative cell viability at $24 \mathrm{~h}, 48 \mathrm{~h}$ and at $96 \mathrm{~h} .10 \mu \mathrm{l}$ of MTT solution was added to $100 \mu \mathrm{l}$ of culture media and incubated for $4 \mathrm{~h}$ at $37^{\circ} \mathrm{C}$, and the optical density was measured at $570 \mathrm{~nm}$. Each experiment was performed in triplicates.

\section{Soft agar colony formation assay}

Anchorage-independent growth was determined by soft agar analysis as follows. $48 \mathrm{~h}$ after transfection, the HEp-2 cells (500 cells per well) were suspended in soft agar medium (RPMI-1640 media containing $0.3 \%$ agar) and seeded onto a base of RPMI1640 media containing $0.5 \%$ agar in six-well plates. Plates were incubated at $37{ }^{\circ} \mathrm{C}, 5 \% \mathrm{CO}_{2}$ in a humidified incubator for 2 weeks. Colonies were counted under a microscopic field at $\times 100$ magnification. Each assay was performed in triplicates.

\section{Flow cytometry analysis}

At $48 \mathrm{~h}$ after transfection with $m i R-21$ ASO or control ASO, the HEp- 2 cells were detached from the plates by trypsin incubation, rinsed with PBS and fixed in $70 \%(\mathrm{v} / \mathrm{v})$ ethanol. The cells were then rehydrated in PBS and incubated with RNase $(100 \mu \mathrm{g} / \mathrm{ml})$ and propidium iodide $(60 \mu \mathrm{g} / \mathrm{ml})$ (Sigma-Aldrich, MO, USA). Cells were analyzed using the FACSCalibur System (BD Biosciences, San Jose, CA, USA), and the cell cycle phase was determined by 
Cell Quest analysis. The proliferation index (PI) was calculated as follows: $\mathrm{PI}=(\mathrm{S}+\mathrm{G} 2 / \mathrm{M}) / \mathrm{G} 1(\mathrm{~S}, \mathrm{G} 2 / \mathrm{M}$ and $\mathrm{G} 1$ refer to the percentage of cells in $\mathrm{S}$ phase, G2/M phase and G1 phase, respectively.) [30].

\section{Cell apoptosis analysis}

HEp-2 cells were transfected with $m i R-21$ ASO or control ASO, and seeded into triplicate wells on a 14-well slide (CelLine/Erie Scientific Co., NH, USA) at 48-h post-transfection. HEp-2 cells cultured in complete medium, including $0.09 \mathrm{mg} / \mathrm{ml}$ teniposide (VM-26), an apoptosis inducer, were taken as positive control. HEp-2 cells cultured only in complete medium were taken as negative control. Cell apoptosis was detected by the In situ Cell Death Detection Kit, Fluorescein (Roche Applied Science, IN, USA) according to the manufacturer's instructions. DAPI staining was used to determine the number of nuclei and to assess gross cell morphology.

\section{In vivo assay}

Female BALB/c athymic nude mice (6-week-old) (Institute of Zoology, Chinese Academy of Sciences) were used for in vivo study. A total of $1 \times 10^{6} \mathrm{HEp}-2$ cells transfected with miR-21 ASO or control ASO, as well as the non-transfected HEp-2 cells, were implanted subcutaneously in the right armpit of nude mice. Each group consisted of six mice. Tumor volumes were measured from the first 6 days, and once every other day. Tumor volume was calculated from (length $\times$ width $\left.^{2}\right) / 2$ and presented as mean \pm standard deviation (SD) $\mathrm{mm}^{3}$ [31]. Statistical analysis was performed using the Student's $t$-test to compare the tumor volume between different treatment groups.

\section{Semi-quantitative RT-PCR}

Total RNAs were extracted from transfected HEp-2 cells for semi-quantitative RT-PCR (sqRT-PCR) at $48 \mathrm{~h}$ post-transfection. Target genes and the endogenous control gene, $\beta$-actin, were included in the same reaction. PCR products were electrophoresed on $1 \%$ agarose gels containing ethidium bromide and visualized under UV transilluminator. Bands were quantified with Labworks 4.0 software.

\section{Western blot}

Total protein from HEp-2 cells transfected with either oligonucleotides or plasmids was extracted using RIPA buffer (1 mM $\mathrm{MgCl}_{2}, 10 \mathrm{mM}$ Tris- $\mathrm{HCl} \mathrm{pH} 7.4,1 \%$ Triton $\mathrm{X}-100,0.1 \%$ SDS, $1 \% \mathrm{NP}-40$ ), and protein expression was analyzed by western blot. $\beta$-Tubulin was served as a loading control. Total protein extracts were separated on $12 \%$ SDS-PAGE gels and transferred to nitrocellulose membranes. The level of BTG2 expression was evaluated using the rabbit polyclonal anti-BTG2 antibody (Saier Biotech, Tianjin, China), which is immunogen affinity purified. As a loading control, the $\beta$-tubulin expression level was measured using rabbit polyclonal anti- $\beta$-tubulin antibody (Sigma-Aldrich, MO, USA). Bands were quantified with Labworks 4.0 software.

\section{EGFP reporter assay}

The HEp-2 cells were seeded in 24-well plates and cotransfected with $700 \mathrm{ng}$ of the fluorescence reporter plasmid, $300 \mathrm{ng}$ of the pDsRed2-N1 plasmid (as a control for transfection efficiency), and $100 \mathrm{pmol}$ of oligonucleotides or $500 \mathrm{ng}$ of the miRNA-expressing construct using Lipofectamine 2000 reagent (Invitrogen). At $48 \mathrm{~h}$ after transfection, EGFP and RFP (red fluorescent protein) activities were measured with the F-4500 fluorescence spectrophotometer (HITACHI, Japan). The EGFP value was normalized by the RFP value.

\section{Statistical analysis}

Most in vitro experiments were performed thrice. Where appropriate, the results are expressed as mean \pm standard deviation (SD) and statistically analyzed using the Student's $t$-test for the comparison of two groups, with $P<0.05$ considered to be significant.

\section{Conflict of Interest}

The authors declare no competing financial interests.

\section{Acknowledgments}

This work was supported by grants from the National Natural Science Foundation of China (No. 30873017) and the Key Program of the Natural Science Foundation of Tianjing (No. 08JCZDJC23300). We thank Tianjin First Center Hospital for providing human laryngeal tissue samples. We also thank the College of Public Health of Tianjin Medical University for the technical assistance in fluorescent detection. The ArrayExpress accession numbers of miRNA microarray design and cDNA microarray design are A-MEXP-1506 and A-MEXP-1511. The ArrayExpress accession numbers of miRNA microarray experiment and cDNA microarray experiment are E-MEXP-2039 and E-MEXP-2056.

\section{References}

1 Lewis BP, Burge CB, Bartel DP. Conserved seed pairing, often flanked by adenosines, indicates that thousands of human genes are microRNA targets. Cell 2005; 120:15-20.

2 Calin GA, Croce CM. MicroRNA signatures in human cancers. Nat Rev Cancer 2006; 6:857-866.

3 Cho WC. OncomiRs: the discovery and progress of microRNAs in cancers. Mol Cancer 2007; 6:60.

4 Kent OA, Mendell JT. A small piece in the cancer puzzle: microRNAs as tumor suppressors and oncogenes. Oncogene 2006; 25:6188-6196.

5 Mertens-Talcott SU, Chintharlapalli S, Li X, Safe S. The oncogenic microRNA-27a targets genes that regulate specificity protein transcription factors and the G2-M checkpoint in MDA-MB-231 breast cancer cells. Cancer Res 2007; 67:11001-11011.

6 le Sage C, Nagel R, Egan DA, et al. Regulation of the p27(Kip1) tumor suppressor by miR-221 and miR-222 promotes cancer cell proliferation. EMBO J 2007; 26:3699-3708.

7 Gillies JK, Lorimer IA. Regulation of $\mathrm{p} 27^{\mathrm{Kip} 1}$ by miRNA 221/222 in glioblastoma. Cell Cycle 2007; 6:2005-2009.

8 Si ML, Zhu S, Wu H, Lu Z, Wu F, Mo YY. MiR-21-mediated tumor growth. Oncogene 2006; 26:2799-2803.

9 Chan JA, Krichevsky AM, Kosik KS. MicroRNA-21 is an antiapoptotic factor in human glioblastoma cells. Cancer Res 2005; 65:6029-6033.

10 Iorio MV, Ferracin M, Liu CG, et al. MicroRNA gene expression deregulation in human breast cancer. Cancer Res 2005; 65:7065-7070. 
11 Zhu S, Wu H, Wu F, Nie D, Sheng S, Mo YY. MicroRNA-21 targets tumor suppressor genes in invasion and metastasis. Cell Res 2008; 18:350-359.

12 Krek A, Grun D, Poy M, et al. Combinatorial microRNA target predictions. Nat Genet 2005; 37:495-500.

13 Lewis BP, Shih I, Jones-Rhoades MW, Bartel DP, Burge CB. Prediction of mammalian microRNA targets. Cell 2003; 115:787-798.

14 Bartel DP. MicroRNAs: genomics, biogenesis, mechanism, and function. Cell 2004; 116:281-297.

15 Meng F, Henson R, Wehbe-Janek H, et al. MicroRNA-21 regulates expression of the PTEN tumor suppressor gene in human hepatocellular cancer. Gastroenterology 2007; 133:647658.

16 Zhu S, Si ML, Wu H, Mo YY. MicroRNA-21 targets the tumor suppressor gene tropomyosin 1 (TPM1). J Biol Chem 2007; 282:14328-14336.

17 Frankel LB, Christoffersen NR, Jacobsen A, et al. Programmed cell death 4 (PDCD4) is an important functional target of the microRNA miR-21 in breast cancer cells. $J$ Biol Chem 2008; 283:1026-1033.

18 Asangani IA, Rasheed SA, Nikolova DA, et al. MicroRNA-21 (miR-21) post-transcriptionally downregulates tumor suppressor Pdcd4 and stimulates invasion, intravasation and metastasis in colorectal cancer. Oncogene 2008; 27:2128-2136.

19 Lim LP, Lau NC, Garrett-Engele P, et al. Microarray analysis shows that some microRNAs downregulate large numbers of target mRNAs. Nature 2005; 433:769-773.

20 Wang X, Wang X. Systematic identification of microRNA functions by combining target prediction and expression profiling. Nucleic Acids Res 2006; 34:1646-1652.

21 Lim IK. TIS21 (/BTG2/PC3) as a link between ageing and cancer: cell cycle regulator and endogenous cell death molecule. J Cancer Res Clin Oncol 2006; 132:417-426.

22 Melamed J, Kernizan S, Walden PD. Expression of B-cell translocation gene 2 protein in normal human tissues. Tissue Cell 2002; 34:28-32.

23 Ficazzola MA, Fraiman M, Gitlin J, et al. Antiproliferative $\mathrm{B}$ cell translocation gene 2 protein is down-regulated posttranscriptionally as an early event in prostate carcinogenesis. Carcinogenesis 2001; 22:1271-1279.

24 Struckmann K, Schraml P, Simon R, et al. Impaired expression of the cell cycle regulator BTG2 is common in clear cell renal cell carcinoma. Cancer Res 2004; 64:1632-1638.

25 Kawakubo H, Carey JL, Brachtel E, et al. Expression of the NF-kappaB responsive gene BTG2 is aberrantly regulated in breast cancer. Oncogene 2004; 23:8310-8319.

26 Guardavaccaro D, Corrente G, Covone F, et al. Arrest of $\mathrm{G}(1)-\mathrm{S}$ progression by the p53-inducible gene PC3 is Rb dependent and relies on the inhibition of cyclin D1 transcription. Mol Cell Biol 2000; 20:1797-1815.

27 Lim IK, Lee MS, Ryu MS, et al. Induction of growth inhibition of 293 cells by downregulation of the cyclin $\mathrm{E}$ and cyclin-dependent kinase 4 proteins due to overexpression of TIS21. Mol Carcinog 1998; 23:25-35.

28 Manjarrez ME, Ocadiz R, Valle L, et al. Gariglio P. Detection of human papillomavirus and relevant tumor suppressors and oncoproteins in laryngeal tumors. Clin Cancer Res 2006; 12:6946-6951.

29 Carleton M, Cleary MA, Linsley PS. MicroRNAs and cell cycle regulation. Cell Cycle 2007; 6:2127-2132.

30 Seifer DB, MacLaughlin DT, Penzias AS, et al. Gonadotropin-releasing hormone agonist-induced differences in granulosa cell cycle kinetics are associated with alterations in follicular fluid müllerian-inhibiting substance and androgen content. J Clin Endocrinol Metab 1993; 76:711-714.

31 Qian SB, Chen SS. Transduction of human hepatocellular carcinoma cells with human alpha-interferon gene via retroviral vector. World J Gastroenterol 1998; 4:210-213. 